\title{
On Plane Motion of Incompressible Variable Viscosity Fluids with Moderate Peclet Number in Presence of Body Force Via Von-Mises Coordinates
}

\author{
Mushtaq Ahmed \\ Department of Mathematics, University of Karachi, Karachi, Pakistan \\ Email address: \\ mushtaqku@yahoo.com, muahmed@uok.edu.pk
}

To cite this article:

Mushtaq Ahmed. On Plane Motion of Incompressible Variable Viscosity Fluids with Moderate Peclet Number in Presence of Body Force Via Von-Mises Coordinates. International Journal of Fluid Mechanics \& Thermal Sciences. Vol. 5, No. 3, 2019, pp. 67-74. doi: $10.11648 /$ j.ijfmts.20190503.12

Received: March 13, 2019; Accepted: July 22, 2019; Published: August 10, 2019

\begin{abstract}
The aim of this article is to use von-Mises coordinates to find a class of new exact solutionsof the equations governing the plane steady motion with moderate Peclet number of incompressible fluid of variable viscosity in presence of body force. An equation relating a differentiable function and a stream function characterizes the class under consideration. When the differentiable function is parabolic and when it is not, in both the cases, it finds exact solutions for given one component of the body force. This discourse shows an infinite set of streamlines and the velocity components, viscosity function, generalized energy function and temperature distribution for moderate Peclet number in presence of body force. Moreover, for parabolic case, it obtains viscosity as a function of temperature distribution for moderate Peclet number.
\end{abstract}

Keywords: Martin's System, Von-Mises Coordinates, Variable Viscosity, Navier-Stokes Equations with Body Force, Exact Solutions with Body Force

\section{Introduction}

In general, a moving fluid element experiences both the surface and body forces. The momentum of moving fluid element is given by the Navier-Stokes equations (NSE). The non-linear terms in NSE offers a great difficulty for its exact solution show ever, some transformation techniques and dimension analysis methods are workable. A variety of techniques/methods and references given there are practical for some exact solutions of NSE without body force [1-6]. Moreover recently Mushtaq A. et. al., applied a new technique for exact solution of variable viscosity fluids without body force term $[7,10]$. Body force term like coriolis force is considered by Giga, Y. et. al. in [8] and Gerbeau, J. et. al. gives a fundamental remark on NSE with body force in [9] where as Mushtaq A. et. al. has applied successive transformation technique for exact solution for flow of incompressible variable viscosity fluids in presence of body force in [11-14].

To achieve the aim of this letter successive transformation technique is applied. According to this method the basic non- dimensional flows equations with body force in Cartesian space $(x, y)$ are transformed into Martin's coordinates $(\phi, \psi)$ then to von-Mises coordinates $(x, \psi)$. In Martin's coordinates, the curvilinear coordinates $(\phi, \psi)$ are such that the coordinate lines $\psi=$ const. are streamlines and the coordinate lines $\phi=$ constant are arbitrary [15]. Whereas in the von-Mises coordinates, the arbitrary coordinate lines of Martin's system is taken along the $x$-axis. Thus, the function $\phi=x$ and stream function $\psi$ of Martin's coordinates as independent variables instead of $y$ and $x$ [16]. Further, the characteristic equation for streamlines of the class of flows under consideration is:

$$
\frac{y-g(x)-n}{m}=\text { const. }
$$

Where $m \neq 0, n$ are constants and a differentiable function is $g(x)$. Without loss of generality the equation (1) implies 


$$
y=g(x)+v(\psi)
$$

where $v(\psi)=m \psi+n$.

Paper's organization is follow: Section (2) givescentral flow equations in non-dimensional form and transforms them into Martin system $(\phi, \psi)$. Section (3) retransforms the basic equations to von-Mises coordinates. The exact solutions to the problem in presence of body force are given in section 4 . Conclusions are given at the end.

\section{Basic Non-dimensional Equations in Martin's Coordinates}

The equation of continuity, NSE and energy equation, for the steady plane motion of incompressible fluid of variable viscosity with constant thermal conductivity in the presence of unknown external force, in non-dimensional form are respectively following

$$
\begin{gathered}
u_{x}+v_{y}=0 \\
u u_{x}+v u_{y}=F_{1}-p_{x} \\
+\frac{1}{\mathrm{R}_{\mathrm{e}}}\left[\left(2 \mu u_{x}\right)_{x}+\left\{\mu\left(u_{y}+v_{x}\right)\right\}_{y}\right] \\
u v_{x}+v v_{y}=F_{2}-p_{y} \\
+\frac{1}{\mathrm{R}_{\mathrm{e}}}\left[\left(2 \mu v_{y}\right)_{y}+\left\{\mu\left(u_{y}+v_{x}\right)\right\}_{x}\right] \\
u T_{x}+v T_{y}=\frac{1}{\mathrm{R}_{\mathrm{e}} \mathrm{P}_{\mathrm{r}}}\left(T_{x x}+T_{y y}\right) \\
+\frac{\mathrm{E}_{\mathrm{c}}}{\mathrm{R}_{\mathrm{e}}}\left[2 \mu\left(u_{x}^{2}+v_{y}^{2}\right)+\mu\left(u_{y}+v_{x}\right)^{2}\right]
\end{gathered}
$$

Where $u(x, y), v(x, y)$ are the components of velocity vector, $F_{1}(x, y), F_{2}(x, y)$ are the components of the body force, $p(x, y)$ pressure, $\mu(x, y)$ the viscosity, and $T(x, y)$ is temperature. The numbers $E_{c}, P_{r}$ and $R_{e}$ are the Ecart number, the Prandtl number and the Reynolds number respectively. The product of $R_{e}$ and $P_{r}$ is Peclet number $P_{e^{\prime}}$. The solution of the basic fluid dynamics equations is found for very large and very small $P_{e^{\prime}}$ where as the solution for moderate $P_{e^{\prime}}$ is challenging. Please refer to [17-22] and reference therein.

The solution of the equation (3) is a stream function $\psi(x, y)$ such that $\frac{\partial^{2} \psi}{\partial x \partial y}=\frac{\partial^{2} \psi}{\partial y \partial x}$ and

$$
u=\frac{\partial \psi}{\partial y}, v=-\frac{\partial \psi}{\partial x}
$$

The solution of the remaining system of equations (4-6), as experience teaches, offers a great difficulty because of the presence of the non-linear term. These equations are managed by introducing the total energy function $T_{x}$ and the vorticity function $\Omega$ defined by:

$$
\begin{gathered}
T_{x}=p+\frac{1}{2}\left(u^{2}+v^{2}\right)-\frac{2 \mu u_{x}}{\mathrm{R}_{\mathrm{e}}} \\
\Omega=v_{x}-u_{y}
\end{gathered}
$$

Utilizing equation (8-9) in equations (4-6), we have

$$
\begin{gathered}
-v \Omega=F_{1}-L_{x}+\frac{1}{\mathrm{R}_{\mathrm{e}}} A_{y} \\
u \Omega=F_{2}-L_{y}-\frac{1}{R_{e}} B_{y}+\frac{1}{\mathrm{R}_{\mathrm{e}}} A_{x} \\
u T_{x}+v T_{y}=\frac{1}{P_{e^{\prime}}}\left(T_{x x}+T_{y y}\right) \\
+\frac{\mathrm{E}_{\mathrm{c}}}{\mathrm{R}_{\mathrm{e}}} \frac{1}{4 \mu}\left(B^{2}+4 A^{2}\right)
\end{gathered}
$$

where

$$
A=\mu\left(u_{y}+v_{x}\right) \text { and } B=4 \mu u_{x}
$$

Consider the allowable change of coordinates:

$$
x=x(\phi, \psi), y=y(\phi, \psi)
$$

where the system $(\phi, \psi)$ are curvilinear coordinates in the $(x, y)$ - plane such that the Jacobian $J=\frac{\partial(x, y)}{\partial(\phi, \psi)} \neq 0$ is finite. Let curvilinear coordinate $\psi$ is stream function as defined in Martin [15]. Let $\lambda$ be the angle between the tangent to the streamlines $\psi=$ const. and the curves $\phi=$ const. as arbitrary at a point $P(x, y)$, then

$$
\tan (\lambda)=\frac{y_{\phi}}{x_{\phi}}
$$

The first fundamental form is

$d s^{2}=E(\phi, \psi) d \phi^{2}+2 F(\phi, \psi) d \phi d \psi+G(\phi, \psi) d \psi^{2}$

wherein:

$$
\begin{gathered}
E=x_{\phi}^{2}+y_{\phi}^{2} \\
F=x_{\phi} x_{\psi}+y_{\phi} y_{\psi} \\
G=\left(x_{\psi}\right)^{2}+\left(y_{\psi}\right)^{2}
\end{gathered}
$$


Differentiating equation (14) with respect to $x$ and $y$, and solving the resulting equations, one finds:

$$
\begin{aligned}
& y_{\phi}=-J \psi_{x}, y_{\psi}=J \phi_{x} \\
& x_{\phi}=J \psi_{y}, x_{\psi}=-J \phi_{y}
\end{aligned}
$$

wherein

$$
J= \pm \sqrt{E G-F^{2}}= \pm\left(x_{\phi} y_{\psi} y_{\phi} x_{\psi}\right)= \pm W
$$

Application of trigonometric identities on equation (15) and equation (18) provides

$$
\begin{gathered}
x_{\phi}=\sqrt{E} \operatorname{Cos}(\lambda) \\
x_{\psi}=\frac{1}{\sqrt{E}}[F \operatorname{Cos}(\lambda)-J \operatorname{Sin}(\lambda)] \\
y_{\phi}=\sqrt{E} \operatorname{Sin}(\lambda) \\
y_{\psi}=\frac{1}{\sqrt{E}}[F \operatorname{Sin}(\lambda)+J \operatorname{Cos}(\lambda)]
\end{gathered}
$$

The integrability conditions:

$$
x_{\psi \phi}=x_{\phi \psi}, y_{\psi \phi}=y_{\phi \psi}
$$

for $x$ and $y$, yield:

$$
\lambda_{\phi}=\frac{J \Gamma_{11}^{2}}{E}, \lambda_{\psi}=\frac{J \Gamma_{12}^{2}}{E}
$$

where

$$
\begin{gathered}
\Gamma_{11}^{2}=\frac{1}{2 W^{2}}\left[-F E_{\phi}+2 E F_{\phi}-E E_{\psi}\right] \\
\Gamma_{12}^{2}=\frac{1}{2 W^{2}}\left[E G_{\phi}-F E_{\psi}\right]
\end{gathered}
$$

Equation (21), applying the integrability condition $\lambda_{\phi \psi}=\lambda_{\psi \phi}$ for $\lambda(\phi, \psi)$, yields

$$
K=\frac{1}{W}\left[\left(\frac{W \Gamma_{11}^{2}}{E}\right)_{\psi}-\left(\frac{W \Gamma_{12}^{2}}{E}\right)_{\phi}\right]
$$

where $\mathrm{K}$ is called the Gaussian curvature.

Now equations (10-11), on substituting equation (15), equation (18), equation (20) andequations (22-23) simplifies to following

$$
\begin{aligned}
& -R_{e} \Omega J E=-F R_{e} J \sqrt{E}\left(F_{1} \cos \lambda+F_{2} \sin \lambda\right) \\
& +J R_{e} J \sqrt{E}\left(F_{1} \sin \lambda-F_{2} \cos \lambda\right)+R_{e} J E L_{\psi}
\end{aligned}
$$

$$
\begin{aligned}
& +A_{\phi}\left(\left(F^{2}-J^{2}\right) \cos 2 \lambda-2 F J \sin 2 \lambda\right) \\
& \left.+E A_{\psi}(J \sin 2 \lambda-F \cos 2 \lambda)\right) \\
& -B_{\phi}\left(\frac{1}{2}\left(F^{2}-J^{2}\right) \sin 2 \lambda+F J \cos 2 \lambda\right) \\
& +E B_{\psi}\left(\frac{1}{2} F \sin 2 \lambda+J \cos ^{2} \lambda\right)
\end{aligned}
$$

and

$$
\begin{gathered}
0=R_{e} J \sqrt{E}\left[F_{1} \cos \lambda+F_{2} \sin \lambda\right] \\
-R_{e} J L_{\phi}+E A_{\psi} \cos 2 \lambda \\
-A_{\phi}[F \cos 2 \lambda-J \sin 2 \lambda] \\
+B_{\phi}\left(\frac{1}{2} F \sin 2 \lambda-J \sin ^{2} \lambda\right)-\frac{E B_{\psi}}{2} \sin 2 \lambda
\end{gathered}
$$

According to differential geometry [23], the expression $u T_{x}+v T_{y}$ in equation (12) simplifies to $\frac{T_{\phi}}{J}$ and

$$
\left(T_{x x}+T_{y y}\right)
$$

$$
=\frac{1}{J}\left[\left(\frac{G T_{\phi}-F T_{\psi}}{J}\right)_{\phi}+\left(\frac{E T_{\psi}-F T_{\phi}}{J}\right)_{\psi}\right]
$$

Therefore, the energy equation (12)becomes

$$
\begin{gathered}
\frac{1}{\mathrm{~J} P_{e^{\prime}}}\left[\left(\frac{G T_{\phi}-F T_{\psi}}{J}\right)_{\phi}+\left(\frac{E T_{\psi}-F T_{\phi}}{J}\right)_{\psi}\right] \\
=-\frac{\mathrm{E}_{\mathrm{c}}}{\mathrm{R}_{\mathrm{e}}} \frac{1}{4 \mu}\left(B^{2}+4 A^{2}\right)+\frac{T_{\phi}}{J}
\end{gathered}
$$

The magnitude of velocity vector $\mathbf{q}=(u, v)$ is $q=\sqrt{u^{2}+v^{2}}$ and it simplifies to:

$$
q=\frac{\sqrt{E}}{J}
$$

The equation (13) on substitute values from equations (1823), provides

$$
\begin{gathered}
B(\phi, \psi)=\frac{4 \mu}{E J^{3}}\left[E_{\phi}(F \sin \lambda+J \cos \lambda)^{2}\right. \\
-2 E(F \sin \lambda+J \cos \lambda)\left(F_{\phi} \sin \lambda+J_{\phi} \cos \lambda\right) \\
\left.+E^{2}\left(J_{\psi} \sin 2 \lambda+G_{\phi} \sin ^{2} \lambda\right)\right]
\end{gathered}
$$




$$
\begin{gathered}
A(\phi, \psi)=\mu\left[-\frac{(F \cos \lambda-J \sin \lambda)}{4 E^{2} J^{5}}\right. \\
\left\{E_{\phi}\left(2 E J^{3} \cos \lambda+F \sqrt{E} \sin \lambda\right)\right. \\
-4 E^{2} J^{2} J_{\phi} \cos \lambda-2 E \sqrt{E} F_{\phi} \sin \lambda \\
\left.+E \sqrt{E} E_{\psi} \sin \lambda\right\} \\
+\frac{\cos \lambda}{2 J^{3}}\left\{E_{\psi}(F \sin \lambda+J \cos \lambda)\right. \\
\left.-2 E J_{\psi} \cos \lambda-E G_{\phi} \sin \lambda\right\} \\
+\frac{(F \sin \lambda+J \cos \lambda)}{2 E J^{3}}\left\{\left(J E_{\phi}-2 E J_{\phi}\right) \sin \lambda\right. \\
\left.+\cos \lambda\left[-F E_{\phi}+2 E F_{\phi}-E E_{\psi}\right]\right\} \\
-\frac{\sin \lambda}{2 J^{3}}\left\{\left(E_{\psi}(J \sin \lambda-F \cos \lambda)\right.\right. \\
\left.\left.-2 E J_{\psi} \sin \lambda+E G_{\phi} \cos \lambda\right\}\right]
\end{gathered}
$$

The vorticity function $\Omega$ in Martin's system is

$$
\Omega=v_{\phi} \phi_{x}+v_{\psi} \psi_{x}-u_{\phi} \phi_{y}-u_{\psi} \psi_{y}
$$

Equation (32) on substituting equation (15), equation (18)and equation (20) provides

$$
\begin{gathered}
\Omega=\frac{(F \sin \lambda+J \cos \lambda)}{2 E J^{3}}\left\{\left(J E_{\phi}-2 E J_{\phi}\right) \sin \lambda\right. \\
\left.+\cos \lambda\left[-F E_{\phi}+2 E F_{\phi}-E E_{\psi}\right]\right\} \\
-\frac{\sin \lambda}{2 J^{3}}\left\{E_{\psi}(J \sin \lambda-F \cos \lambda)\right. \\
\left.\left.-2 E J_{\psi} \sin \lambda+E G_{\phi} \cos \lambda\right\}\right] \\
+\frac{(F \cos \lambda-J \sin \lambda)}{4 E^{2} J^{5}} \\
\left\{E_{\phi}\left(2 E J^{3} \cos \lambda+F \sqrt{E} \sin \lambda\right)\right. \\
-4 E^{2} J^{2} J_{\phi} \cos \lambda-2 E \sqrt{E} F_{\phi} \sin \lambda \\
\left.+E \sqrt{E} E_{\psi} \sin \lambda\right\} \\
-\left[\frac { \operatorname { c o s } \lambda } { 2 J ^ { 3 } } \left\{E_{\psi}(F \sin \lambda+J \cos \lambda)-2 E J_{\psi} \cos \lambda\right.\right. \\
\left.\left.-E G_{\phi} \sin \lambda\right\}\right]
\end{gathered}
$$

The fundamental system of equations transformed to Martin's system as momentum equations (25-26), the energy equation (28) for moderate Peclet number together with equations (30-31) and equation (33).

\section{Basic Equations in Von-Mises Coordinates}

Since the purpose of this communication is to determine a class of exact solutions to flow equations in von-Mises coordinates therefore the definition of von-Mises coordinates in [16] demands to set

$$
\phi=x
$$

The equation (15), equation (17), equations (19), equations (30-31) and equation (33) reduces to

$$
\begin{gathered}
\cos \lambda=\frac{1}{\sqrt{E}} \\
E=1+\left(x g^{\prime}(x)\right)^{2} \\
J=m x \\
B=\frac{-4 \mu}{m x^{2}} \\
A=\frac{\mu}{m x^{2}}\left(x\left(x g^{\prime}(x)\right)^{\prime}-2 x g^{\prime}(x)\right)
\end{gathered}
$$

and

$$
\Omega=\frac{\left(x g^{\prime}(x)\right)^{\prime}}{m x}
$$

The equations (25-26) and equation (28) on utilizing equations (34-40), give

$$
\begin{gathered}
-R_{e} \Omega=-R_{e}\left(m x F_{2}\right)+R_{e} L_{\psi}-m x A_{x} \\
+x g^{\prime} A_{\psi}+B_{\psi} \\
0=R_{e}\left(F_{1}+x g^{\prime} F_{2}\right) \\
-R_{e} L_{x}+\frac{A_{\psi}\left(1-\left(x g^{\prime}\right)^{2}\right)}{m x}+x g^{\prime} A_{x}-\frac{x g^{\prime} B_{\psi}}{m x} \\
m x T_{x x}-2 x g^{\prime} T_{\psi x}+\frac{\left(1+\left(x g^{\prime}\right)^{2}\right)}{m x} T_{\psi \psi} \\
+\left(m-P_{e^{\prime}}\right) T_{x}+\left(x g^{\prime}\right)^{\prime} T_{\psi}
\end{gathered}
$$




$$
=-\frac{m \times E_{c} P_{r}}{4 \mu}\left(B^{2}+4 A^{2}\right)
$$

and

$$
q=\frac{\sqrt{1+N^{2}}}{m x}, N=x g^{\prime}(x)
$$

Applying the integrability condition $L_{x \psi}=L_{\psi x}$ on equations (41-42) yields

$$
\begin{gathered}
m x A_{x x}-2 N A_{x \psi}-\frac{\left[1-N^{2}\right]}{m x} A_{\psi \psi}+m A_{x} \\
-A_{\psi} N^{\prime}-\left\{B_{x}-\frac{g^{\prime} B_{\psi}}{a}\right\}_{\psi}=R_{e} \Omega_{x} \\
+R_{e}\left(F_{1}+F_{2} N\right)_{\psi}-R_{e}\left(m x F_{2}\right)_{x}
\end{gathered}
$$

Through the solution of equation (45), the viscosity $\mu$ from either equation (38) or equation (39), the generalized energy function $L$ from equations (41-42)for pressure $p$ from Equation (9), the temperature distribution $T$ for moderate Peclet number from equation (43), the velocity components from equation (7)streamlines from equation (2) are found.

\section{Exact Solutions in Presence of Body \\ Force}

The compatibility equation (45) involves functions $A$ and $B$ which depends upon the viscosity function $\mu, g(x)$ and derivative of $g(x)$ and the body force components $F_{1}, F_{2}$. Although the analytical solution of equation (45) extremely difficult however the difficulty is eased on eliminating on eliminating $\mu$ from equation (38) and equation (39) on introducing the function $X(x)$ as follow

$$
A=X(x) B
$$

where

$$
X(x)=\frac{-1}{4}\left(x N^{\prime}-2 N\right)
$$

provided $\left(x N^{\prime}-2 N\right) \neq 0$.

Use of equation (46) in equation (45), gives

$$
\begin{gathered}
m x X B_{x x}-(1+2 N X) B_{x \psi} \\
+\frac{B_{\psi \psi}}{m x}\left\{N-X\left(1-N^{2}\right)\right\}+m B_{x}\left\{2 x X^{\prime}+X\right\} \\
-B_{\psi}\left(2 N X^{\prime}+N^{\prime} X\right)+m B\left(x X^{\prime}\right)^{\prime}
\end{gathered}
$$

$$
=R_{e}\left(\frac{N^{\prime}}{m x}\right)^{\prime}+R_{e}\left(F_{1}+N F_{2}\right)_{\psi}-R_{e}\left(m x F_{2}\right)_{x}
$$

In equation (48) the coefficients of the derivative $B_{x x}$, $B_{x \psi}, B_{\psi \psi}, B_{x}, B_{\psi}$ and $B$ are all functions of $x$ only, this suggests to seek a solution of equation(48) of the form

$$
B(x, \psi)=R(x)+S(\psi)
$$

Equation (48), on substituting equation (49), becomes

$$
\begin{gathered}
{\left[x(X R)^{\prime}\right]^{\prime}+\frac{S^{\prime \prime}}{m x}\left\{N-X\left(1-N^{2}\right)\right\}} \\
-S^{\prime}\left(2 N X^{\prime}+N^{\prime} X\right)+m\left(x X^{\prime}\right)^{\prime} S \\
=R_{e}\left(\frac{N^{\prime}}{m x}\right)^{\prime}+R_{e}\left(F_{1}+N F_{2}\right)_{\psi}-R_{e}\left(m x F_{2}\right)_{x}
\end{gathered}
$$

The equation (50) involves the components of unknown body force $F_{1}(x, \psi), F_{2}(x, \psi)$ the functions $R(x)$ and $S(\psi)$ therefore the solution of equation (50) will depend upon the form of $F_{1}$ and $F_{2}$. One select many possible forms of $F_{1}$ and $F_{2}$ leading to the solution of equation (50) for $R(x)$ and $S(\psi)$, however they are required to satisfy (41-42) and (43). The search for the appropriate form of $F_{1}$ or $F_{2}$ reveals

$$
R_{e}\left(m x F_{2}\right)_{x}=R_{e}\left(\frac{N^{\prime}}{m x}\right)^{\prime}-\left[x(X R)^{\prime}\right]^{\prime}
$$

or

$$
R_{e} F_{2}=R_{e}\left(\frac{N^{\prime}}{m^{2} x^{2}}\right)-(X R)^{\prime}+\frac{Q_{1}(\psi)}{m x}
$$

where $Q_{1}(\psi)$ is function of integration.

Insertion of equation (52) in equation (50) keeps $R(x)$ and $S(\psi)$ arbitrary and provides

$$
\begin{gathered}
R_{e} F_{1}=\frac{S^{\prime}}{m x}\left\{N-X\left(1-N^{2}\right)\right\} \\
-S\left(2 N X^{\prime}+N^{\prime} X\right)+m\left(x X^{\prime}\right) \int S d \psi \\
+P_{1}(x)-N\left[R_{e}\left(\frac{N^{\prime}}{m^{2} x^{2}}\right)-(X R)^{\prime}+\frac{Q_{1}(\psi)}{m x}\right]
\end{gathered}
$$

where $P_{1}(x)$ is function of integration. Solution of equations (41-42) for $L$, on substituting equations (52-53), is following 


$$
\begin{gathered}
R_{e} L=\int Q_{1}(\psi) d \psi+m\left(x X^{\prime}\right) \int S(\psi) d \psi \\
-(N X+1) S(\psi)+e^{x} \int e^{-x} N(X R)^{\prime} d x+e^{x} c_{1}
\end{gathered}
$$

provided

$$
m=1
$$

$c_{1}$ is constant of integration.

Thus from equation (38) or equation (39)viscosity is

$$
\mu=-\frac{x^{2}}{4}[R(x)+S(\psi)]
$$

The energy equation (43), on utilizing equation (46), equation (49) and equations (55-56) becomes

$$
\begin{gathered}
x T_{x x}-2 N T_{\psi x}+\frac{\left(1+N^{2}\right)}{x} T_{\psi \psi}+\left(1-P_{e^{\prime}}\right) T_{x}-N^{\prime} T_{\psi}= \\
E_{c} P_{r} \frac{\left(1+4 X^{2}\right)}{x}\{R(x)+S(\psi)\}
\end{gathered}
$$

The right-hand side of equation (57) suggests seeking solution of the form

$$
T(x, \psi)=T_{1}(x)+T_{2}(x) H(\psi)
$$

Equation (57) for equation (58) becomes

$$
\begin{aligned}
& \left.x T_{1}^{\prime \prime}+\left(1-P_{e^{\prime}}\right) T_{1}^{\prime}\right\}+H\left\{x T_{2}^{\prime \prime}+\left(1-P_{e^{\prime}}\right) T_{2}^{\prime}\right\} \\
& +H^{\prime}\left\{-2 N T_{2}^{\prime}-N^{\prime} T_{2}\right\}+\frac{\left(1+N^{2}\right) T_{2}}{x} H^{\prime \prime} \\
& =E_{c} P_{r} \frac{\left(1+4 X^{2}\right)}{x}\{R(x)+S(\psi)\}
\end{aligned}
$$

Let us differentiate equation (59) with respect to $\psi$.

$$
\begin{aligned}
& H^{\prime}(\psi)\left\{x T_{2}^{\prime \prime}+\left(1-P_{e^{\prime}}\right) T_{2}^{\prime}\right\}+H^{\prime \prime}(\psi)\left\{-2 N T_{2}^{\prime}-N^{\prime} T_{2}\right\} \\
& +\frac{\left(1+N^{2}\right) T_{2}}{x} H^{\prime \prime \prime}(\psi)=E_{c} P_{r} \frac{\left(1+4 X^{2}\right)}{x} S^{\prime}(\psi)
\end{aligned}
$$

Since $x$ and $\psi$ are independent variables therefore the right-hand side of equation (60) demands

$$
S(\psi)=s_{1} \psi+s_{2}
$$

and

$$
H(\psi)=s_{3} \psi+s_{4}
$$

where $s_{1}, s_{2}, s_{3}$ and $s_{4}$ are constants of integration. Substitution of equations (61-62) in equation (60)provides

$$
x T_{2}^{\prime \prime}+\left(1-P_{e^{\prime}}\right) T_{2}^{\prime}=\frac{E_{c} P_{r} s_{1}}{s_{3}} \frac{\left(1+4 X^{2}\right)}{x}
$$

Utilization of equations(61-62) in equation(59), gives

$$
\begin{gathered}
x T_{1}^{\prime \prime}+\left(1-P_{e^{\prime}}\right) T_{1}^{\prime}=s_{3}\left\{2 N T_{2}^{\prime}+N^{\prime} T_{2}\right\} \\
-s_{4}\left\{x T_{2}^{\prime \prime}+\left(1-P_{e^{\prime}}\right) T_{2}^{\prime}\right\}
\end{gathered}
$$

$$
+E_{c} P_{r} \frac{\left(1+4 X^{2}\right)}{x}\left\{R(x)+s_{2}\right\}
$$

when $\left(1-P_{e^{\prime}}\right) \neq 0$ the solution of equations (63-64) are

$$
\begin{aligned}
& T_{2}(x)=\int\left\{x^{-\left(1-P_{e^{\prime}}\right)} \int x^{\left(1-P_{e^{\prime}}\right)} Z_{2}(x) d x\right\} d x \\
&+c_{2} \int x^{-\left(1-P_{e^{\prime}}\right)} d x+c_{3} \\
& T_{1}(x)=\int\left\{x^{-\left(1-P_{e^{\prime}}\right)} \int x^{\left(1-P_{e^{\prime}}\right)} Z_{1}(x) d x\right\} d x \\
&+c_{4} \int x^{-\left(1-P_{e^{\prime}}\right)} d x+c_{5}
\end{aligned}
$$

where $c_{2}, c_{3}, c_{4}$ and $c_{5}$ are constant of integration and

$$
\begin{gathered}
Z_{2}(x)=\frac{E_{c} P_{r} s_{1}\left(1+4 X^{2}\right)}{s_{3} x^{2}} \\
Z_{1}(x)=s_{3}\left\{2 N T_{2}^{\prime}+N^{\prime} T_{2}\right\} \\
-s_{4}\left\{x T_{2}^{\prime \prime}+\left(1-P_{e^{\prime}}\right) T_{2}^{\prime}\right\} \\
+E_{c} P_{r} \frac{\left(1+4 X^{2}\right)}{x}\left\{R(x)+s_{2}\right\}
\end{gathered}
$$

Utilization of equations (65-68) in equation (58) provides the temperature $T$ formoderate $P_{e^{\prime}}$ and the back substitution gives the viscosity $\mu$ from equation (56), the velocity components from equation (7), the pressure $p$ from equation (9) using equation (55), and streamlines from equation (2) for non-parabolic function $g(x)$.

Now when $\left(1-P_{e^{\prime}}\right)=0$ the equations $(65-66)$ give

$$
T_{2}(x)=\int\left\{\int Z_{2}(x) d x\right\} d x+c_{6} x+c_{7}
$$

and

$$
T_{1}(x)=\int\left\{\int Z_{3}(x) d x\right\} d x+c_{8} x+c_{9}
$$

where

$$
Z_{3}(x)=s_{3}\left\{2 N T_{2}^{\prime}+N^{\prime} T_{2}\right\}-s_{4} x T_{2}^{\prime \prime}
$$




$$
+E_{c} P_{r} \frac{\left(1+4 X^{2}\right)}{x}\left\{R(x)+s_{2}\right\}
$$

and $c_{6}, c_{7}, c_{8}$ and $c_{9}$ are constants of integration. Insertion of equations (69-71) in equation (58) gives $T$ for

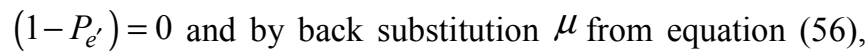
the velocity components from equation (7), $p$ from equation (9) using equation (56), and streamlines from equation (2) for non-parabolic function $g(x)$.

The case $\left(x N^{\prime}-2 N\right)=0$, on supplying $N(x)$ from equation (35), shows that the function $g(x)$ is a parabolic function

$$
g(x)=\frac{1}{2} c_{10} x^{2}+c_{11}
$$

where $c_{10}$ and $c_{11}$ are constants. In view of equation (72), the equation (48) reduces to

$$
\begin{gathered}
-B_{x \psi}+\frac{N B_{\psi \psi}}{m x} \\
=R_{e}\left(F_{1}+N F_{2}\right)_{\psi}-R_{e}\left(m x F_{2}\right)_{x}
\end{gathered}
$$

Here equation (73) is to provide the function $B(x, \psi)$ but it involves the components of unknown body force $F_{1}(x, \psi)$ and $F_{2}(x, \psi)$ therefore its solution will depend upon the form of $F_{1}$ and $F_{2}$. It is easy to see that the arbitrary selection of the forms for $F_{1}$ and $F_{2}$ to the solution of equation (73) for $B(x, \psi)$ does not lead to the solution of the momentum equations (41-42) for the function $L$ and the energy equation (43) for $T$. However, it is found that the solution of the equations (41-42) is obtainable if the function $F_{2}$ is a solution of the following differential equation

$$
R_{e}\left(m x F_{2}\right)_{x}=0
$$

or

$$
R_{e} F_{2}=\frac{Q_{2}(\psi)}{m x}
$$

where a function of integration is $Q_{2}(\psi)$. Substitution of equation (75) in equation (73), provides

$$
R_{e} F_{1}=-\frac{c_{10} x}{m} Q_{2}(\psi)-B_{x}+\frac{c_{10} x}{a} B_{\psi}+P_{2}(x)
$$

where the function of integration is $P_{2}(x)$. Utilizing equations (75-76), in equations (41-42) and solving for the function $L$ one have

$$
\begin{gathered}
R_{e} L=-R_{e}\left(\frac{2 c_{10}}{m}\right) \psi+\int Q_{2}(\psi) d \psi-B(x, \psi) \\
+\int P_{2}(x) d x
\end{gathered}
$$

In view of equation (72), the energy equation (43), becomes

$$
\begin{gathered}
m x^{2} T_{x x}-2 c_{10} x^{3} T_{\psi x}+\frac{1}{m}\left(1+c_{10}^{2} x^{4}\right) T_{\psi \psi} \\
+x\left(m-P_{e^{\prime}}\right) T_{r}+2 c_{10} x^{2} T_{\psi}=E_{c} P_{r} B(x, \psi)
\end{gathered}
$$

On substituting value from equation (43) in equation (78), the viscosity $\mu$ is obtained as a function of temperature $T$

$$
\begin{gathered}
\mu=\left(\frac{-m x^{2}}{4 E_{c} P_{r}}\right)\left[m x^{2} T_{x x}-2 c_{10} x^{3} T_{\psi x}\right. \\
+\frac{1}{m}\left(1+c_{10}^{2} x^{4}\right) T_{\psi \psi}+x\left(m-P_{e^{\prime}}\right) T_{r} \\
\left.+2 c_{10} x^{2} T_{\psi}\right]
\end{gathered}
$$

for moderate $P_{e^{\prime}}$. It is now easy to find the velocity components from equation (7), the pressure $p$ from equation (9) using equation (77), and streamlines from equation (2) for $g(x)$ given by equation (72).

\section{Conclusion}

The following dimensionless parameters are used to obtain the non-dimensional form of the basic equations for the twodimensional steady motion of incompressible fluid of variable viscosity in the presence of body force

$$
\begin{aligned}
& x^{*}=\frac{x}{L_{0}}, y^{*}=\frac{y}{L_{0}}, u^{*}=\frac{u}{U_{0}}, v^{*}=\frac{v}{U_{0}} \\
& \mu^{*}=\frac{\mu}{\mu_{0}}, p^{*}=\frac{p}{p_{0}}, F_{1}^{*}=\frac{F_{1}}{F_{0}}, F_{2}^{*}=\frac{F_{2}}{F_{0}}
\end{aligned}
$$

where $c_{v}=c_{p}=$ Const . where $c_{v}$ is specific heat at constant volume and $c_{p}$ is specific heat at constant pressure, the thermal conductivity $k=k_{0}=$ Const . and density $\rho=\rho_{0}=$ Const.

This paper finds a class of new exact solutions of the equations governing the two-dimensional steady motion with moderate Peclet number of incompressible fluid of variable viscosity in presence of body force in von-Mises coordinates. The characteristic equation for the streamlinesis $y=g(x)+\psi+n$ where a differentiable function is $g(x)$,

$\psi$ is stream function and $n$ is constant. The exact solutions for moderate Peclet number in the presence of body force is 
determined for given one component of the body force, for both the cases when $g(x)$ is non-parabolic function and when it is a parabolic function of $x$. For non-parabolic $g(x)$ the streamlines are $y-g(x)-n=\psi=$ Const. and for parabolic case $g(x)=\frac{1}{2} c_{10} x^{2}+c_{11}$ and the streamlines are $\left[y-\frac{1}{2} c_{10} x^{2}+c_{11}-n\right]=\psi=$ Const. where $c_{10}$ and $c_{11}$ are constants. In both the cases, an infinite set of velocity components, viscosity function, generalized energy function, temperature distribution for moderate Peclet number in presence of body force can be constructed and graph of streamlines can easily be drawn through computer algebra system software to observe the streamline patterns. For parabolic case, viscosity is obtained as a function of temperature distribution for moderate Peclet number.

\section{References}

[1] Chandna, O. P., Oku-Ukpong E. O.; Flows for chosen vorticity functions-Exact solutions of the Navier-Stokes Equations: International Journal of Applied Mathematics and Mathematical Sciences, 17 (1) (1994) 155-164.

[2] Naeem, R. K.; Exact solutions of flow equations of an incompressible fluid of variable viscosity via one - parameter group: The Arabian Journal for Science and Engineering, 1994, 19 (1), 111-114.

[3] Naeem, R. K.; Srfaraz, A. N.; Study of steady plane flows of an incompressible fluid of variable viscosity using Martin's System: Journal of Applied Mechanics and Engineering, 1996, $1(1), 397-433$.

[4] Naeem, R. K.; Steady plane flows of an incompressible fluid of variable viscosity via Hodograph transformation method: Karachi University Journal of Sciences, 2003, 3(1), 73-89.

[5] Naeem, R. K.; On plane flows of an incompressible fluid of variable viscosity: Quarterly Science Vision, 2007, 12 (1), 125-131.

[6] Landau L. D. and Lifshitz E. M.; Fluid Mechanics, Pergmaon Press, vol 6.

[7] Naeem, R. K.; Mushtaq A.; A class of exact solutions to the fundamental equations for plane steady incompressible and variable viscosity fluid in the absence of body force: International Journal of Basic and Applied Sciences, 2015, 4(4), 429-465.www.sciencepubco.com/index.php/IJBAS, doi:10.14419/ijbas.v4i4.5064.

[8] Giga, Y.; Inui, K.; Mahalov; Matasui S.; Uniform local solvability for the Navier-Stokes equations with the Coriolis force: Method and application of Analysis, 2005, 12, 381-384.

[9] Gerbeau, J. -F.; Le Bris, C., A basic Remark on Some NavierStokes Equations With Body Forces: Applied Mathematics Letters, 2000, 13(1), 107-112.

[10] Mushtaq A., On Some Thermally Conducting Fluids: Ph. D Thesis, Department of Mathematics, University of Karachi,
Pakistan, 2016.

[11] Mushtaq A.; Naeem R. K.; S. Anwer Ali; A class of new exact solutions of Navier-Stokes equations with body force for viscous incompressible fluid,: International Journal of Applied Mathematical Research, 2018, 7 (1), 22-26. www.sciencepubco.com/index.php/IJAMR, doi: 10.14419/ijamr.v7i1.8836.

[12] Mushtaq Ahmed, Waseem Ahmed Khan: A Class of New Exact Solutions of the System of PDE for the plane motion of viscous incompressible fluids in the presence of body force,: International Journal of Applied Mathematical Research, 2018, 7 (2), 42-48. www.sciencepubco.com/index.php/IJAMR, doi: 10.14419 /ijamr.v7i2.9694.

[13] Mushtaq Ahmed, Waseem hmed Khan, S. M. Shad Ahsen:A Class of Exact Solutions of quations for Plane Steady Motion of Incompressible Fluids of ariable viscosity in presence of ody Force,: International Journal of Applied Mathematical $\begin{array}{llll}\text { Research, } & 2018, & 7 & \text { (3), }\end{array}$ www.sciencepubco.com/index.php/IJAMR, doi: 10.14419/ijamr.v7i2.12326.

[14] Mushtaq Ahmed, (2018), A Class of New Exact Solution of equations for Motion of Variable Viscosity Fluid In presence of Body Force with Moderate Peclet number, International Journal of Fluid Mechanics and Thermal Sciences, 4 (4) 429www.sciencepublishingdroup.com/j/ijfms 10.11648/j.ijfmts.20180401.12.

[15] Martin, M. H.; The flow of a viscous fluid I: Archive for Rational Mechanics and Analysis, 1971, 41 (4), 266-286.

[16] Daniel Zwillinger; Handbook of differential equations; Academic Press, Inc. (1989).

[17] R. Kronig and J. C. Brink, On the Theory of Extraction from Falling Droplets, Appl. Sci. Res. Ser. A, 2: 142-154, 1950.

[18] B. Abramzon and C. Elata, Numerical analysis of unsteady conjugate heat transfer between a single spherical particle and surrounding flow at intermediate Reynoldsand Peclet numbers, 2nd Int. Conf. on numerical methods in Thermal problems, Venice, pp. 1145-1153, 1981.

[19] D. L. R. Oliver \& K. J. De Witt, High Peclet number heat transfer from adroplet suspended in an electric field: Interior problem, Int. J. Heat Mass Transfer, vol. 36: 3153-3155, 1993.

[20] Z. G. Feng, E. E. Michaelides, Unsteady heat transfer from a spherical particle atfinite Peclet numbers, J. Fluids Eng. 118: 96-102, 1996.

[21] Z. G. Fenz, E. E. Michaelides, Unsteady mass transport from a sphere immersedin a porous medium at finite Peclet numbers, Int. J. Heat Mass Transfer 42:3529-3531, 1999.

[22] Fayerweather Carl, Heat Transfer From a Droplet at Moderate Peclet Numbers with heat Generation. PhD. Thesis, U of Toledo, May 2007.

[23] Weatherburn C. E., Differential geometry of three dimensions, Cambridge University Press, 1964. 